\title{
Performance Analysis of Iteratively Decoded Convergent Source Mapping with Sphere Packing-Assisted Differential Space-Time Spreading Technique for Efficient Video Transmission
}

\author{
Ishtiaque Ahmed $\mathbb{D}^{1},{ }^{1}$ Nasru Minallah $\mathbb{D},{ }^{2}$ Jaroslav Frnda $\mathbb{D}^{3},{ }^{3}$ and Jan Nedoma $\mathbb{D}^{4}$ \\ ${ }^{1}$ National Centre in Big Data and Cloud Computing, University of Engineering and Technology Peshawar (NCBC-UETP), \\ Peshawar 25000, Pakistan \\ ${ }^{2}$ Department of Computer Systems Engineering, University of Engineering and Technology Peshawar, Peshawar 25000, Pakistan \\ ${ }^{3}$ Department of Quantitative Methods and Economic Informatics, \\ Faculty of Operation and Economics of Transport and Communications, University of Zilina, Zilina 010 26, Slovakia \\ ${ }^{4}$ Department of Telecommunications, Faculty of Electrical Engineering and Computer Science, \\ VSB-Technical University of Ostrava, Ostrava-Poruba, Czech Republic
}

Correspondence should be addressed to Ishtiaque Ahmed; ishtiaqueahmed2018@gmail.com

Received 10 August 2021; Revised 2 December 2021; Accepted 11 December 2021; Published 29 December 2021

Academic Editor: Lucia Valentina Gambuzza

Copyright (c) 2021 Ishtiaque Ahmed et al. This is an open access article distributed under the Creative Commons Attribution License, which permits unrestricted use, distribution, and reproduction in any medium, provided the original work is properly cited.

\begin{abstract}
With the substantial growth in number of wireless devices, future communication demands overarching research to design highthroughput and efficient systems. We propose an intelligent Convergent Source Mapping (CSM) approach incorporating Differential Space-Time Spreading (DSTS) technique with Sphere Packing (SP) modulation. The crux of CSM process is assured convergence by attaining an infinitesimal Bit-Error Rate (BER). Data Partitioning (DP) H.264 video codec is deployed to gauge the performance of our intelligent and efficient system. For the purpose of efficient and higher data rates, we have incorporated compression efficient source encoding along with error resiliency and transmission robustness features. The proposed system follows the concept of iterations between the Soft-Bit Source-Decoder (SBSD) and Recursive Systematic Convolutional (RSC) decoder. Simulations of the DSTS-SP-assisted CSM system are presented for the correlated narrowband Rayleigh channel, using different CSM rates but constant overall bit-rate budget. The SP-assisted DSTS systems are mainly useful in decoding algorithms that operate without requiring Channel State Information (CSI). The effects of incorporating redundancy via different CSM schemes on the attainable performance and convergence of the proposed system are investigated using EXtrinsic Information Transfer (EXIT) charts. The effectiveness of the proposed system is demonstrated through IT++ based proof-of-concept simulations. The Peak Signal-to-Noise Ratio (PSNR) analysis shows that using Rate-2/6 CSM with minimum Hamming distance $\left(d_{H, \text { min }}\right)$ of 4 offers about $5 \mathrm{~dB}$ gain, compared to an identical overall system code rate but with Rate- $2 / 3$ CSM and $d_{H, \text { min }}$ of 2 . Furthermore, for a consistent value of $d_{H \text {, min }}$ and overall rate, the Rate-2/3 CSM scheme beats the Rate-5/6 CSM by about $2 \mathrm{~dB}$ at the PSNR degradation point of $2 \mathrm{~dB}$. Moreover, the proposed system with Rate- $2 / 3 \mathrm{CSM}$ scheme furnishes an $E_{b} / N_{0}$ gain of $20 \mathrm{~dB}$ when compared with the uniform-rate benchmarker. Clearly, we can say that higher $d_{H \text {, min }}$ and lower CSM values are favourable for our proposed setup.
\end{abstract}

\section{Introduction}

Evolution in Internet of Things (IoT) and recent developments in ultrafast cellular technologies resulted in a constriction of available bandwidth $[1,2]$. It is estimated that escalation in the demand of higher data rates and bandwidth efficient systems will continue to grow with the advent of Fifth-Generation (5G) wireless technology. 5G is expected to revolutionize the outlook of mankind in medical [3] and intelligent communication technologies [4]. Thus, further researches need to be carried out to better cope with the future demands of wireless and multimedia services. 
Traditionally, source coding standards and channel coding schemes have been incorporated in multimedia systems for the transmission of voice and video streams. Source coding is an important approach to compress the original stream of data, focusing on the removal of redundancy from the stream for transmission over bandlimited channels. The unwanted concomitant is that removal of redundancy results in the hostile happening of unreliability in the transmitted data. The main reason for such unreliability is that transmission over a nonideal (practical) channel triggers distortions in the transmitted signal. Very few or no-redundant bits schemes neither detect nor correct any potential error arising from unreliable transmissions. Therefore, multimedia streams present an exacting research topic for wireless channels [5]. To address the issue of unreliability, channel coding is utilized. Channel coding focuses on the addition of redundant bit(s) to make the transmission scheme reliable. Consequently, the concept of Joint Source and Channel Decoding (JSCD), making the most of residual redundancy phenomenon, has remarkably attracted wide attention [6].

The approach of Soft-Bit Source-Decoder (SBSD) was proposed [7] and effectively deployed in Iterative Source and Channel Decoding (ISCD) [8] for better convergence. More specifically, SBSD operates by gleaning the residual redundancy in the source coded stream for yielding extrinsic information, thus transforming the stream into short frames. The authors in [9] proposed two techniques, namely, I-frame and P-frame error concealment methods, for robust video transmission over noisy channels. Several other methods are presented in [5] to overcome the exigent tasks of susceptible transmission and channel errors due to the limited residual redundancy and predictive coding. Advanced Video Coding (AVC) or H.264 video codec is currently the most popular compression standard among the researchers for mobile video systems. The strategy of Data Partitioning (DP) [10] is advantageously subsumed in the H.264 standard, reducing the unfriendly channel effects corrupting the data stream. DP splits the stream in line with the level of significance of data into three substreams, each having specific level of importance. Another approach known as Irregular Variable Length Coding (IrVLC) was efficiently utilized in schemes with joint source and channel coding [11]. In short, researchers continuously strive and have put persistent efforts to hone the overall wireless communication infrastructure by making it incrementally closer to Shannon's capacity [12] pinnacle.

Multiple-Input Multiple-Output (MIMO) system technologies are growingly becoming popular in bandwidth efficient systems supporting ultrafast rates. Such technologies will be of great significance in the future wireless systems such as $5 \mathrm{G}$ and $6 \mathrm{G}$ [13]. One of the prestigious classes of MIMO systems is Space-Time Block Codes (STBCs), offering fairly acceptable performance with a much easier approach of encoding and spreading $[14,15]$. Space-Time Spreading (STS) technique was presented by Hochwald et al. [16] attaining the best possible transmit diversity gain. The authors in [17] developed an orthogonal transmit diversity technique using Sphere Packing (SP) modulation and demonstrated that the SP-assisted STBC exceeds the conventional STBCs in performance. The STBC and STS approaches rely mainly on the technique of coherent detection, which in turn requires Channel State Information (CSI) at the receiver [18]. Besides this, CSI burdens the overall system which dissipates power due to the enormous transmission of training symbols. An alternate to CSI based systems, known as Differential Space-Time Block Coding (DSTBC), was proposed by Tarokh et al. for the cases of two and more transmit antennas $[19,20]$. DSTBC greatly reduced the complexity but the price paid was a slight loss in performance. SP-assisted DSTS approach was deployed to enhance the performance of adaptive multirate wideband speech coding [21], Irregular Variable Length Coding with iterative detection [22, 23], cooperative communication [24], self-concatenated coding [25], and turbo detection [18] systems. The performance analysis of convergent and nonconvergent two-stage DSTS-SP schemes based on video resolution and motion contents was done in [26]. The three-stage performance investigation of compressed video using SP modulation was done for the combinational gain technique of layered steered space-time coding in [27], whereas that for the noncoherent DSTS scheme was presented in [28].

In this letter, we apply the technique of DSTS-SP to CSM and investigate its effects on the Bit-Error Rate (BER) performance and EXtrinsic Information Transfer (EXIT) curves. The main contributions of our research work are as follows:

(i) An instructive preamble to the concept of CSM and its linkage with RSC, devised for attaining assured convergence.

(ii) Incorporating the technique of DSTS-SP in CSM scheme and investigating its effects using two Transmitters (Txs) and a simple Receiver (Rx) with no CSI requirements

(iii) Analysing the effects of alterations in CSM rates and Hamming distance $\left(d_{H, \min }\right)$ on the EXIT convergence and, hence, attainable performance of the proposed system

(iv) Quantifying the video performance of the presented system with the H.264 standard

The remainder of this article is organized as follows. Section 2 gives details about the parametric parlance used in this discourse. An overview of the DSTS-SP based CSM approach is provided in Section 3. The proposed system with the parameter settings is further described and made plain in Section 4. Simulated results are discussed in Section 5. We succinctly conclude the article in Section 6 .

\section{Parametric Terminologies}

Logarithmic-Likelihood Ratio (LLR) or L-value is an important parameter based on the Soft Decision (SD) output and is mainly used to estimate the reliability of received data. In an SD process, the messages are represented in conditional probabilities of their occurrence, such that the 


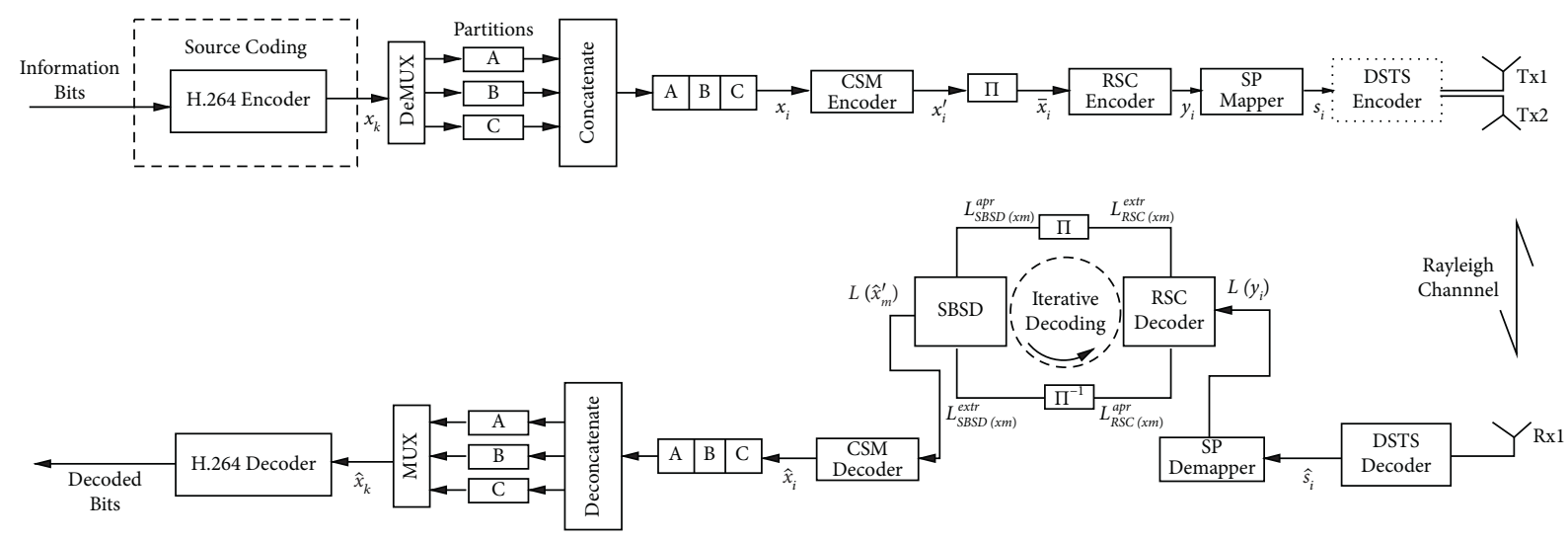

FIGURE 1: Block diagram of the proposed DSTS-SP aided iterative JSCD system.

TABle 1: Proposed DSTS-SP-assisted CSM system parameters.

\begin{tabular}{lccc}
\hline Parameters & Value & Parameters & Value \\
\hline Source code & H.264/AVC & Channel code & CSM \\
Source bit rate & $64 k b p s$ & Tx antennas & 2 \\
Video sequence & QCIF Akiyo & Rx antennas & 1 \\
Frame rate & $15 \mathrm{fps}$ & RSC generator & $\left(G_{0}, G_{1}, G_{2}, G_{3}=13,15,15,17\right)_{8}$ \\
Slices per frame & 9 & Interleaving bits & 10000 \\
Number of MBs per slice & 11 & Normalised Doppler frequency & 0.01 \\
Intraframe MB update & 3 & Modulation scheme & SP \\
MIMO scheme & DSTS & Spreading code & Walsh code \\
\hline
\end{tabular}

received bit is marked either 1 or 0 . The LLR is defined as in the following equation [29]:

$$
L_{X}(u)=\ln \left(\frac{P_{X}(u=0)}{P_{X}(u=1)}\right) .
$$

Here, $L_{X}(u)$ is the LLR or soft value of a random variable $X$ and $P_{X}(u)$ represents the probability of $X$ for its two legitimate values.

One of the key solutions for future ultrafast wireless communication is the MIMO technology. MIMO concept mainly asks for utilizing multiple antennas at base stations to serve a vast range of users connected via single receiving antenna or multiple receiving antennas installed in their devices. There arise some issues like hardware complexity, channel estimation, and correlation of the multiple antennas [30], when considering MIMO schemes. DSTS technique offers a good solution to the issues in MIMO systems and advocates the employment of MIMO schemes for attaining rich diversity gains. Spreading codes are utilized in the systems having MIMO. An STS technique with two transmitters is considered in the proposed system of this treatise. STS algorithm basically splits the data stream into multiple substreams (equal to the number of transmitters) and each substream is transmitted by an assigned antenna. Walsh codes are widely exploited as spreading codes in wireless MIMO schemes, specifically in synchronized multiuser systems. Walsh codes are based on the mutually orthogonal Hadamard codes, providing frequency diversity and very good BER performance.

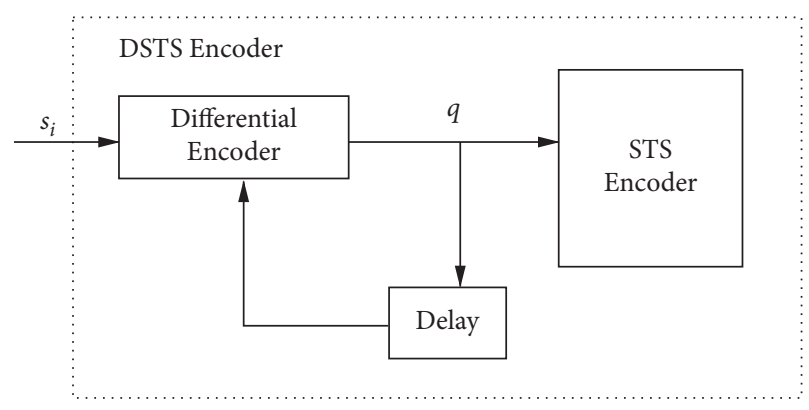

FIgURE 2: Schematic diagram of the DSTS encoder.

Hadamard matrix is necessarily a square matrix generating Walsh code by the continuous conversion between $\mathrm{ON}$ and OFF (1 and -1$)$ states at a specified regular transition interval [31]. Details about the operation and generation of different length Walsh codes using Hadamard matrix are discussed in [32].

SP modulation is scaling up in the construction of errorcorrection codes. Su et al. introduced the merger of transmit diversity techniques with SP modulation, evincing that the SP aided STBC surpassed the conventional STBCs in performance [18]. The viable profit of orthogonal transmit diversity is mostly evaluated using the minimum Euclidean distance [18]. SP modulation results in the best possible minimum Euclidean distance for the symbols, boosting the system's error resilience property. Regarding Euclidean distance, it is simply the length of straight-line segment connecting two coordinates. Hamming distance 
TABLE 2: Different combinations of Mapping-I and Mapping-II schemes.

\begin{tabular}{lcc}
\hline Input symbols & Mapping-I scheme & Mapping-II scheme \\
\hline$S_{(1)}, S_{(2)} . S\left(\begin{array}{c}k \\
2\end{array}\right)$ & $r_{1} b_{1} b_{2} \ldots b_{k}, r_{2} b_{1} b_{2} \ldots b_{k}, r_{2^{K}} b_{1} b_{2} \ldots b_{k}$ & $r_{1} b_{1} b_{2} \ldots b_{k} b_{k} \ldots b_{2} b_{1} r_{1}, r_{2} b_{1} b_{2} \ldots b_{k} b_{k} \ldots b_{2} b_{1} r_{2}, r_{2^{K}} b_{1} b_{2} \ldots b_{k} b_{k} \ldots b_{2} b_{1} r_{2^{K}}$ \\
\hline
\end{tabular}

TABLE 3: CSM-coded symbols with $d_{H \text {, min }}$ and corresponding code rates of constituent encoders.

\begin{tabular}{|c|c|c|c|c|c|}
\hline \multirow{2}{*}{$\begin{array}{l}\text { Error protection } \\
\text { scheme }\end{array}$} & \multirow{2}{*}{ Symbols in decimal } & \multirow{2}{*}{$d_{H, \min }$} & \multicolumn{3}{|c|}{ Code rate } \\
\hline & & & CSM & RSC & Overall \\
\hline $\begin{array}{l}\text { Rate-1 } \\
\text { (benchmarker) }\end{array}$ & 0,1 & 1 & 1 & $1 / 4$ & $1 / 4$ \\
\hline $\begin{array}{l}\mathrm{CSM}_{2}^{3}, \mathrm{CSM}_{3}^{4}, \mathrm{CSM}_{4}^{5} \\
\mathrm{CSM}_{5}^{6}\end{array}$ & $\begin{array}{c}0,3,5,6,0,3,5,6,9,10,12,15,0,3,5,6,9,10,12,15,17,18,20,23, \\
24,27,29,30,0,3,5,6,9,10,12,15,17,18,20,23,24,27,29,30,33, \\
\quad 34,36,39,40,43,45,46,48,51,53,54,57,58,60,63\end{array}$ & $\begin{array}{l}2,2,2, \\
2\end{array}$ & $\begin{array}{c}2 / 3,3 / 4,4 / \\
5,5 / 6\end{array}$ & $\begin{array}{c}3 / 8,1 / 3,5 / \\
16,3 / 10\end{array}$ & $\begin{array}{l}1 / 4,1 / 4,1 / \\
\quad 4,1 / 4\end{array}$ \\
\hline $\begin{array}{l}\operatorname{CSM}_{2}^{6}, \operatorname{CSM}_{3}^{8}, \operatorname{CSM}_{4}^{10} \\
\operatorname{CSM}_{5}^{12}\end{array}$ & $\begin{array}{c}0,30,45,51,0,60,90,102,153,165,195,255,0,120,180,204,306, \\
330,390,510,561,585,645,765,771,891,951,975,0,240,360,408, \\
612,660,780,1020,1122,1170,1290,1530,1542,1782,1902,1950, \\
2145,2193,2313,2553,2565,2805,2925,2973,3075,3315,3435, \\
3483,3687,3735,3855,4095\end{array}$ & $\begin{array}{c}4,4,4 \\
4\end{array}$ & $\begin{array}{c}2 / 6,3 / 8,4 / \\
10,5 / 12\end{array}$ & $\begin{array}{l}3 / 4,2 / 3,1 / \\
\quad 4,3 / 5\end{array}$ & $\begin{array}{l}1 / 4,1 / 4,1 / \\
4,1 / 4\end{array}$ \\
\hline
\end{tabular}

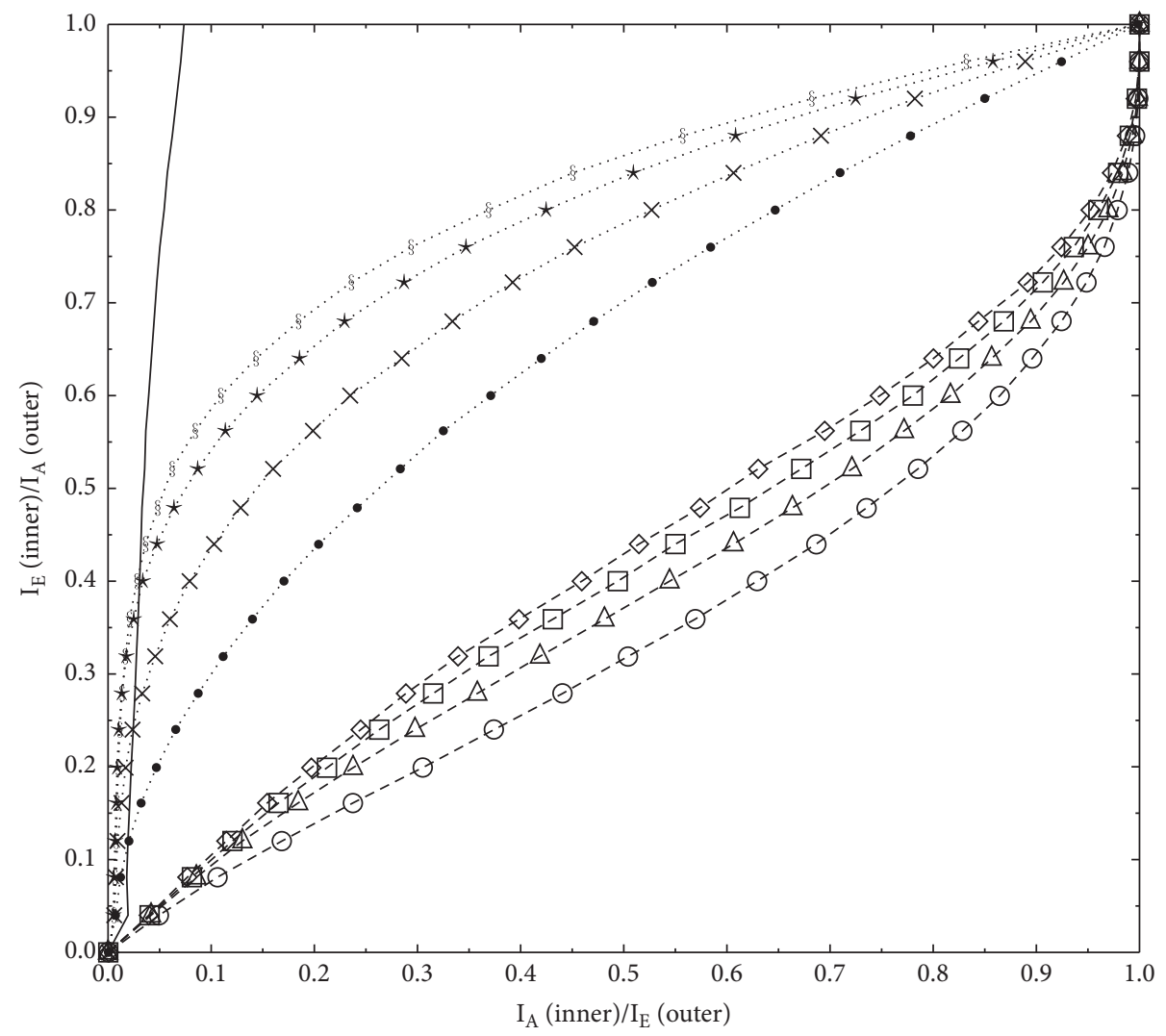

OUTER EXIT CURVES

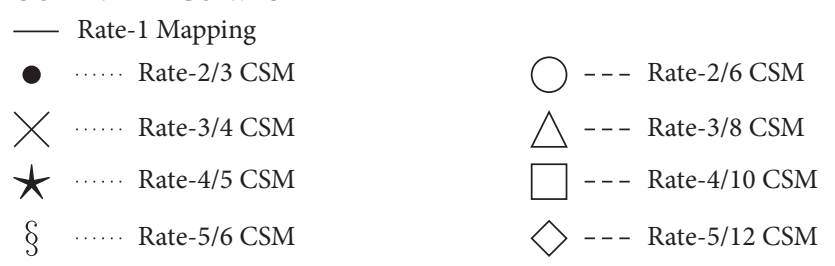

FIgURE 3: EXIT curves for error protection schemes employing different outer (CSM) and inner (RSC) rates. 


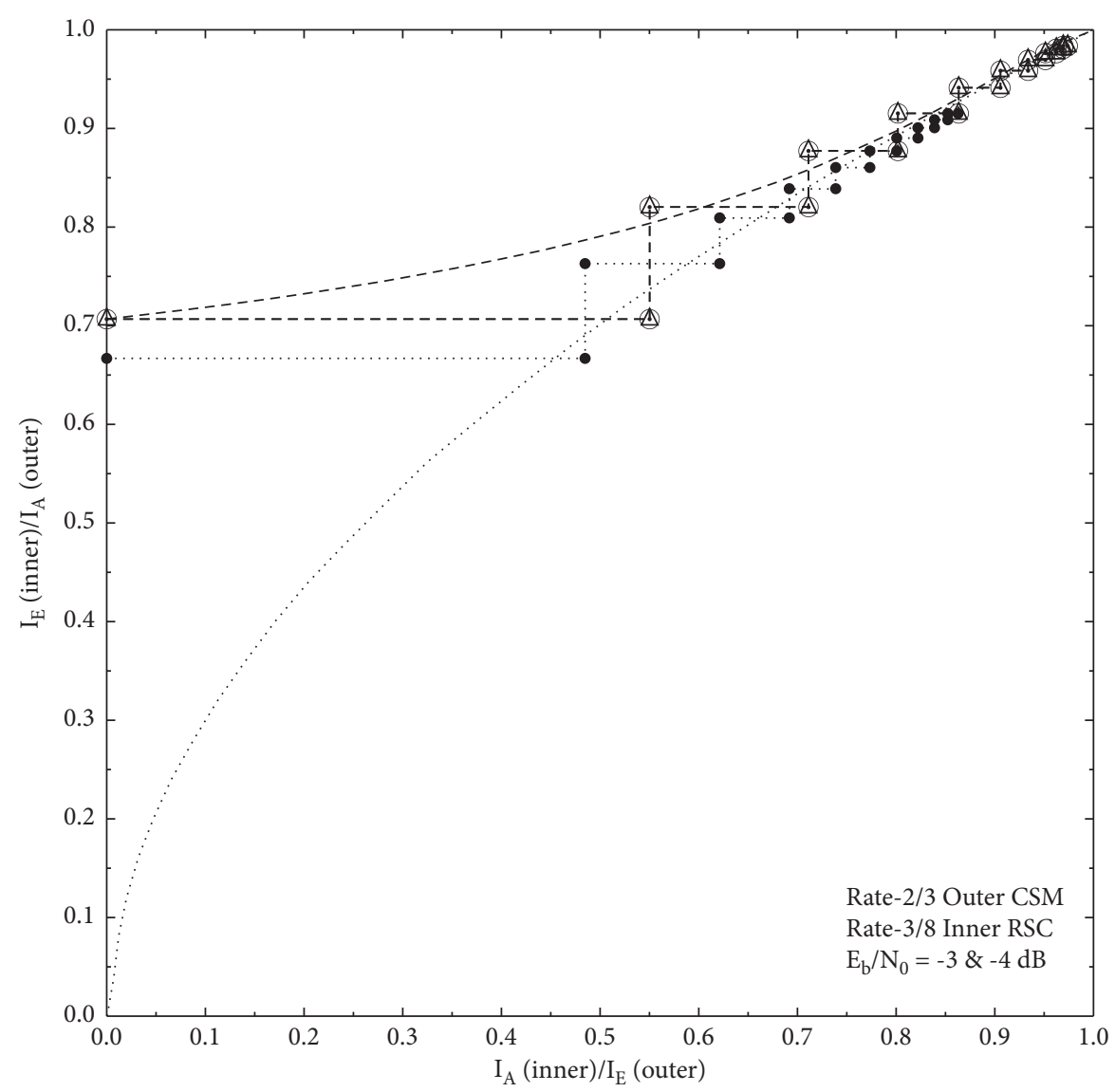

Figure 4: EXIT chart curves and decoding trajectories for Rate-2/3 CSM (Mapping-I) scheme.

terminology, proposed by Hamming [33], is frequently used in the realm of error-correcting codes. Hamming distance quantifies and corrects the emerging error due to noisy channel when data bits are transmitted to the destination. More specifically, Hamming distance shows the difference in two strings with same dimension, that is, the number of varying places in two strings (bitwise exclusive OR, commonly known as XOR) [34]. For example, 10110100 and 10000111 differ in 4 bit places and hence a Hamming distance of 4 exists between them. Similarly, "ROSE" and "NOSE" are single character apart, so a Hamming distance of 1 applies here.

EXIT chart analysis, proposed by Stephen ten Brink [35], promptly predicts the convergence behaviour of iteratively decoded systems. EXIT curves are based on mutual information exchange between the constituent decoders of iterative systems [36]. EXIT charts serve as a handy replacement to the cumbersome Monte Carlo based simulation, offering accurate results for attaining infinitesimal BER [37]. Some common terminologies associated with an EXIT analysis are a priori Information $\left(I_{A}\right)$, a posteriori LLR, and Extrinsic Information $\left(I_{E}\right)$. As given in [37], $I_{A}$ is the already known intrinsic knowledge of any bit and its value is independent of the iterative decoding operation. When any decoder accepts input in the form of LLRs from channel and $I_{A}$, the decoder's output is termed as a posteriori LLR. Performing a mathematical operation of subtracting $I_{A}$ from the first output of concerned decoder yields $I_{E}$. It is worth mentioning that $I_{E}$ can be manipulated with the aid of interleavers and deinterleavers, converting it to $I_{A}$ for other input instances to decoder. EXIT chart analysis asks for two factors in order to generate accurate results $[37,38]$. Firstly, the a priori LLRs should be greatly uncorrelated by utilizing higher interleaving bits and, secondly, the probability density function (PDF) of such LLRs must be Gaussian distribution. The following relations as given in [37] present a clear view of the concepts and terminologies linked with an EXIT analysis.

$$
\begin{gathered}
0 \leq I_{A} \leq 1, \\
0 \leq I_{E} \leq 1, \\
I_{E}=T\left(I_{A}, \frac{E_{b}}{N_{0}}\right), \\
T(0) \leq I_{E} \leq T(1) .
\end{gathered}
$$

According to equations (2) and (3), the values of $I_{A}$ and $I_{E}$ range from 0 to $1 . I_{A}$ to $I_{E}$ conversion requires a transfer function Toperating at specific $E_{b} / N_{0}$, as given in equation (4). The inverse of Texists on the range specified in equation (5). 


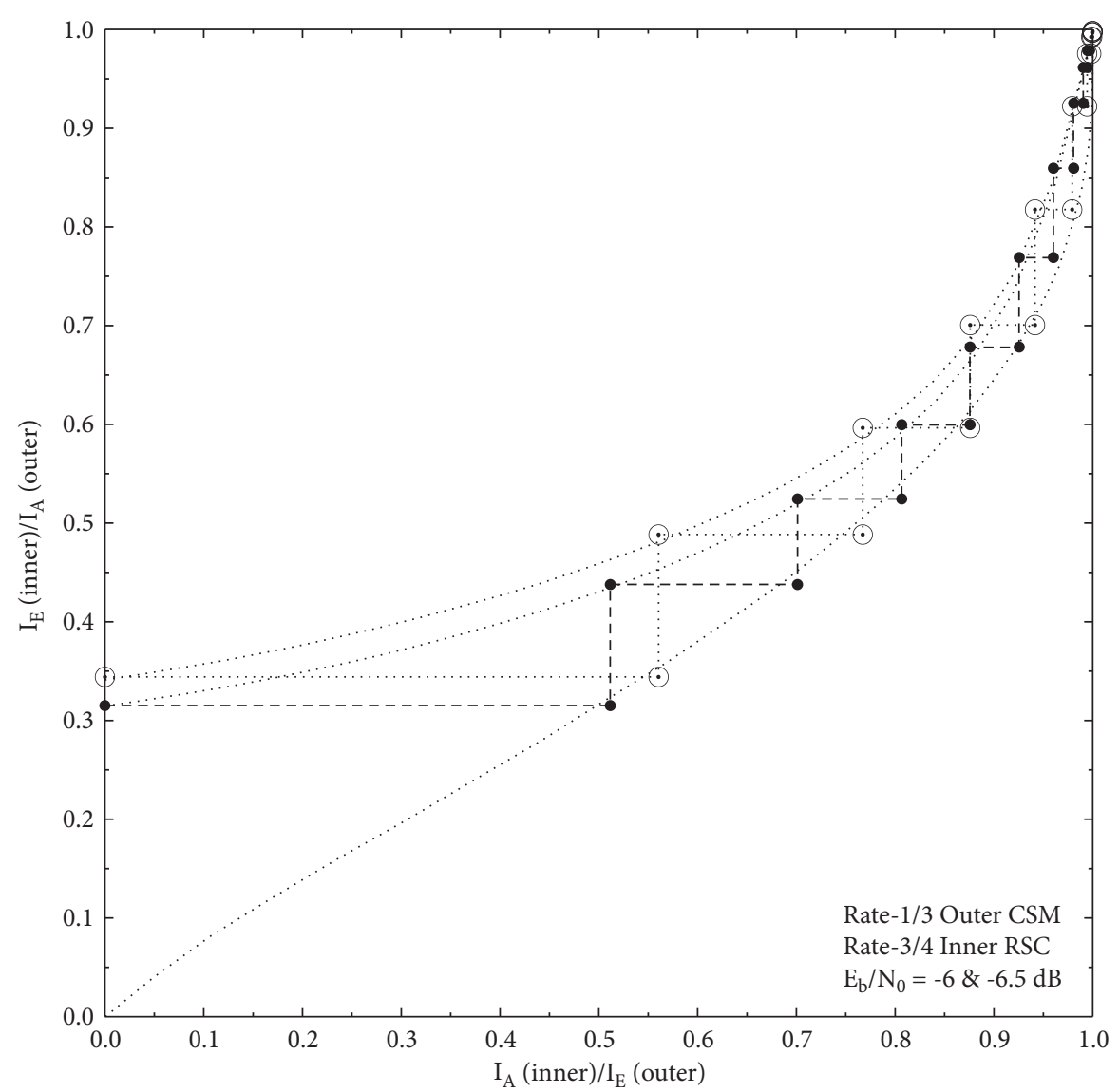

Figure 5: EXIT chart curves and decoding trajectories for Rate-2/6 CSM (Mapping-II) scheme.

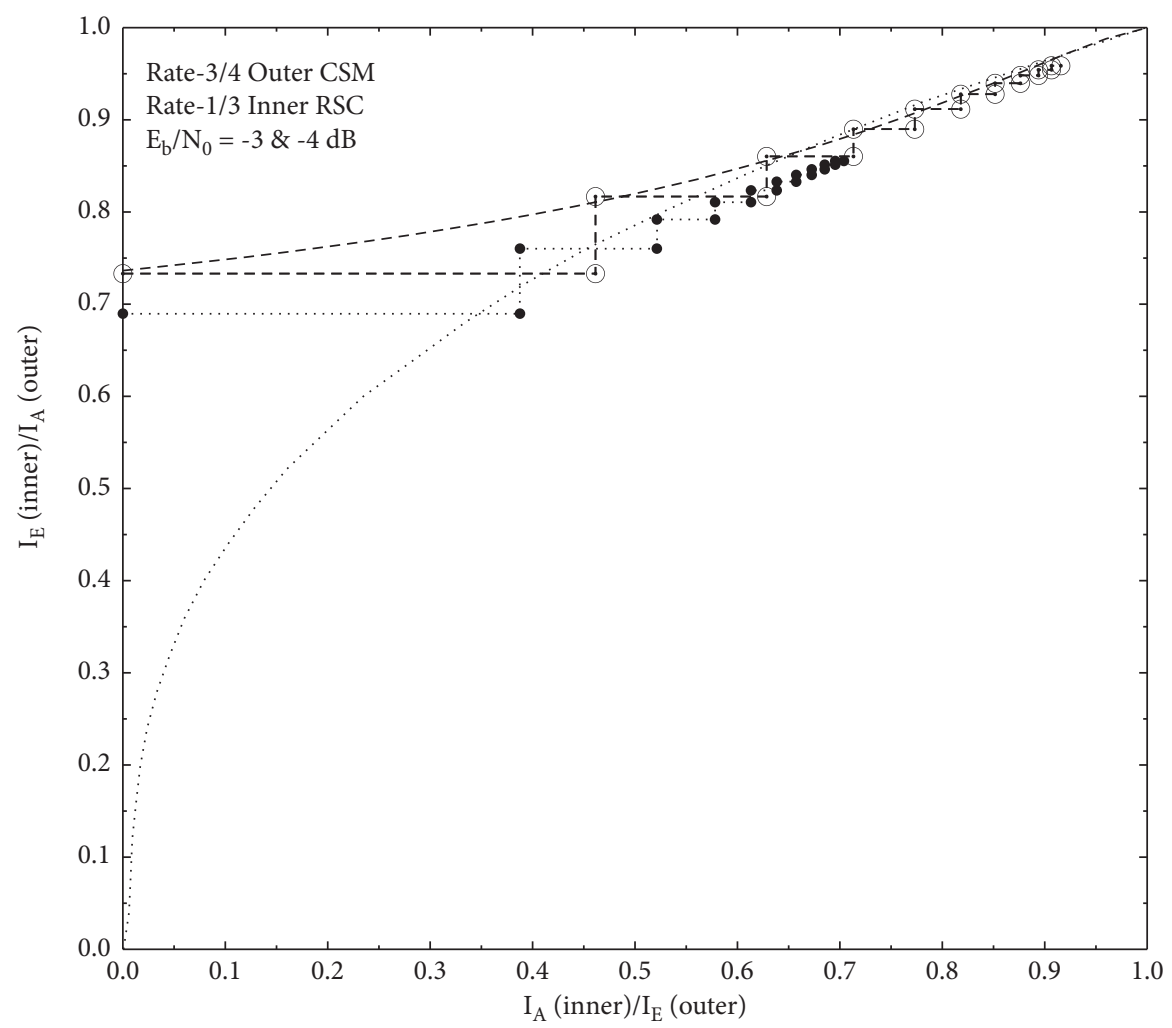

FIgURE 6: EXIT chart curves and decoding trajectories for Rate-3/4 CSM (Mapping-I) scheme. 


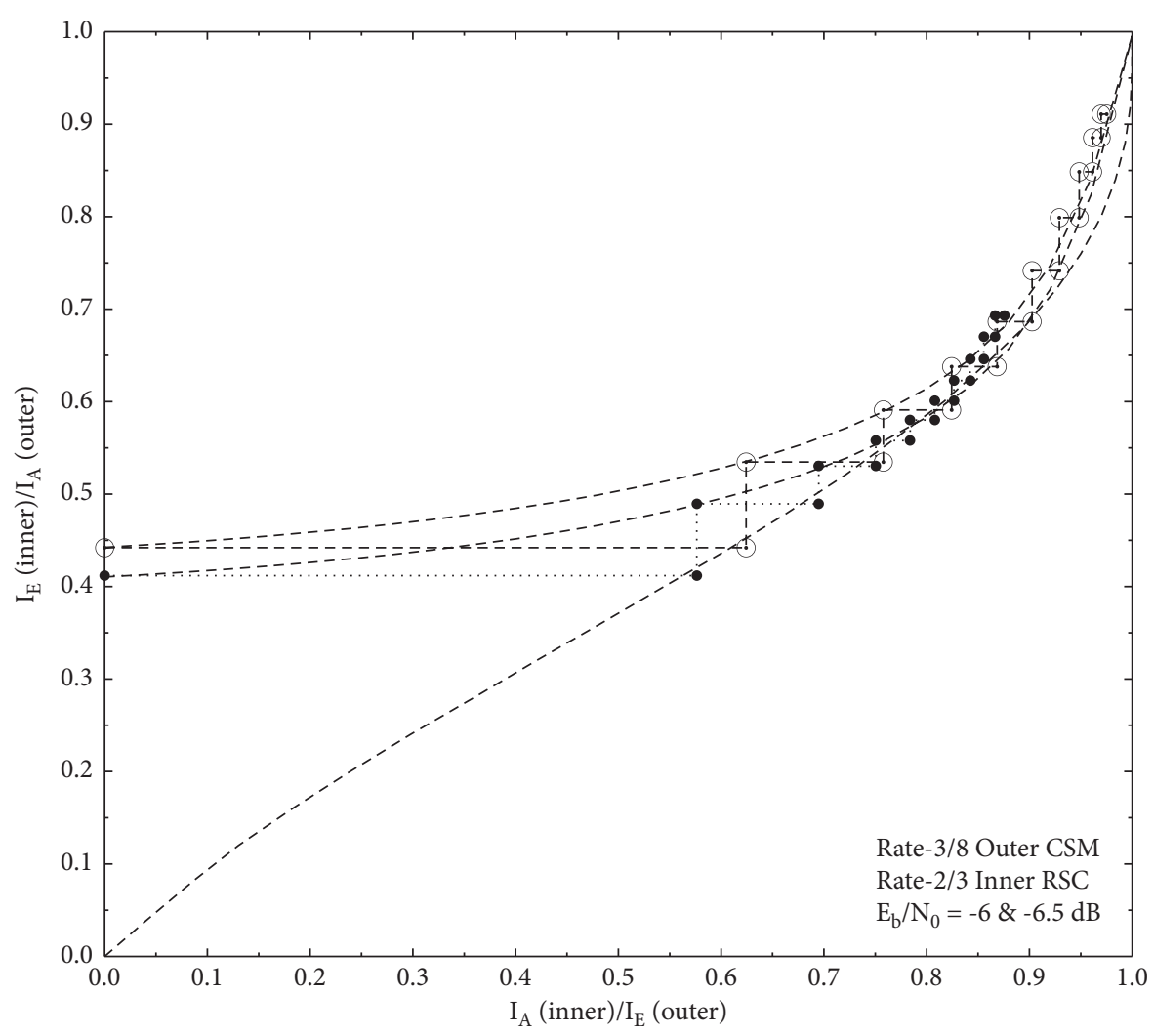

FIgURE 7: EXIT chart curves and decoding trajectories for Rate-3/8 CSM (Mapping-II) scheme.

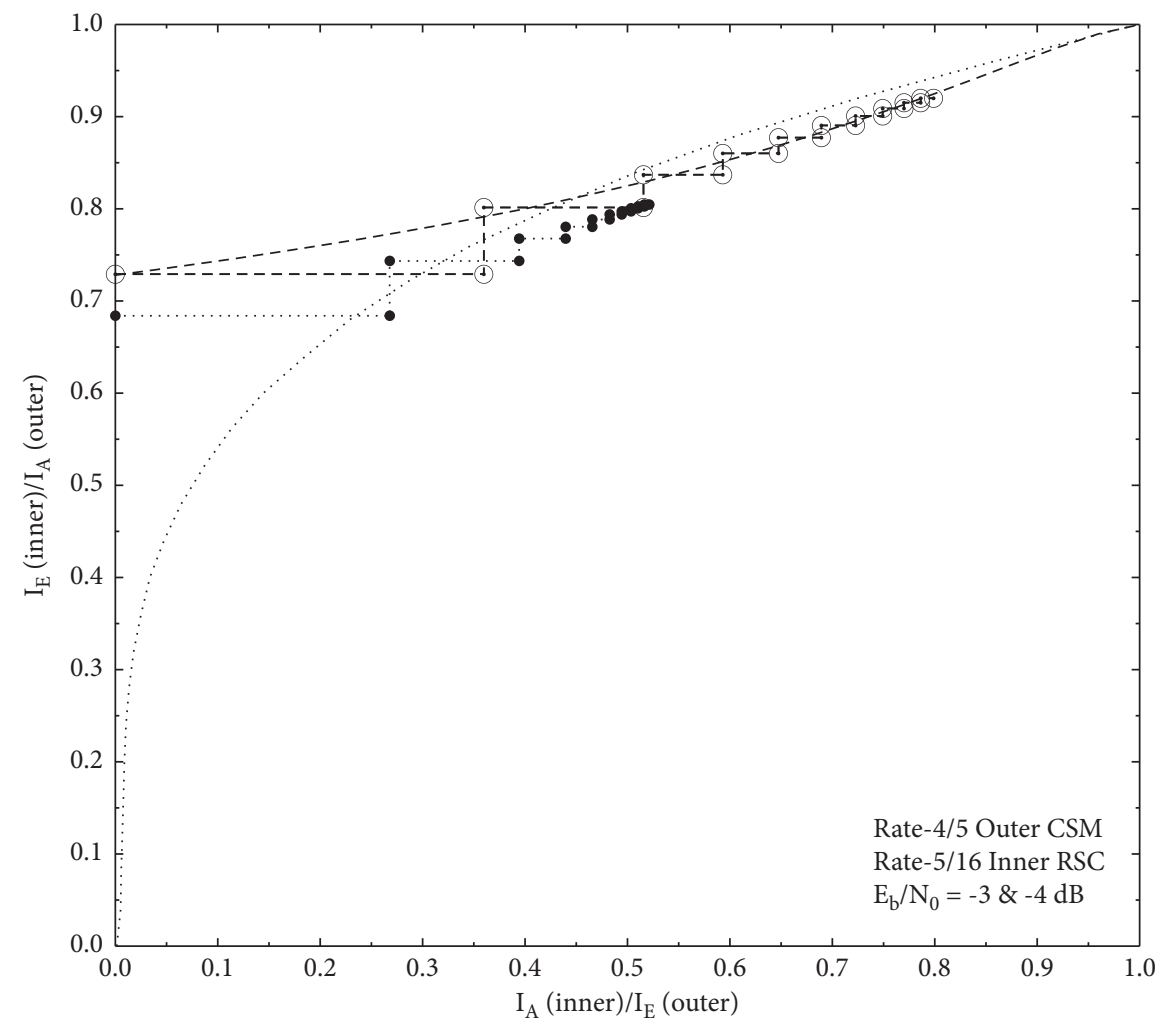

FIgURE 8: EXIT chart curves and decoding trajectories for Rate-4/5 CSM (Mapping-I) scheme. 


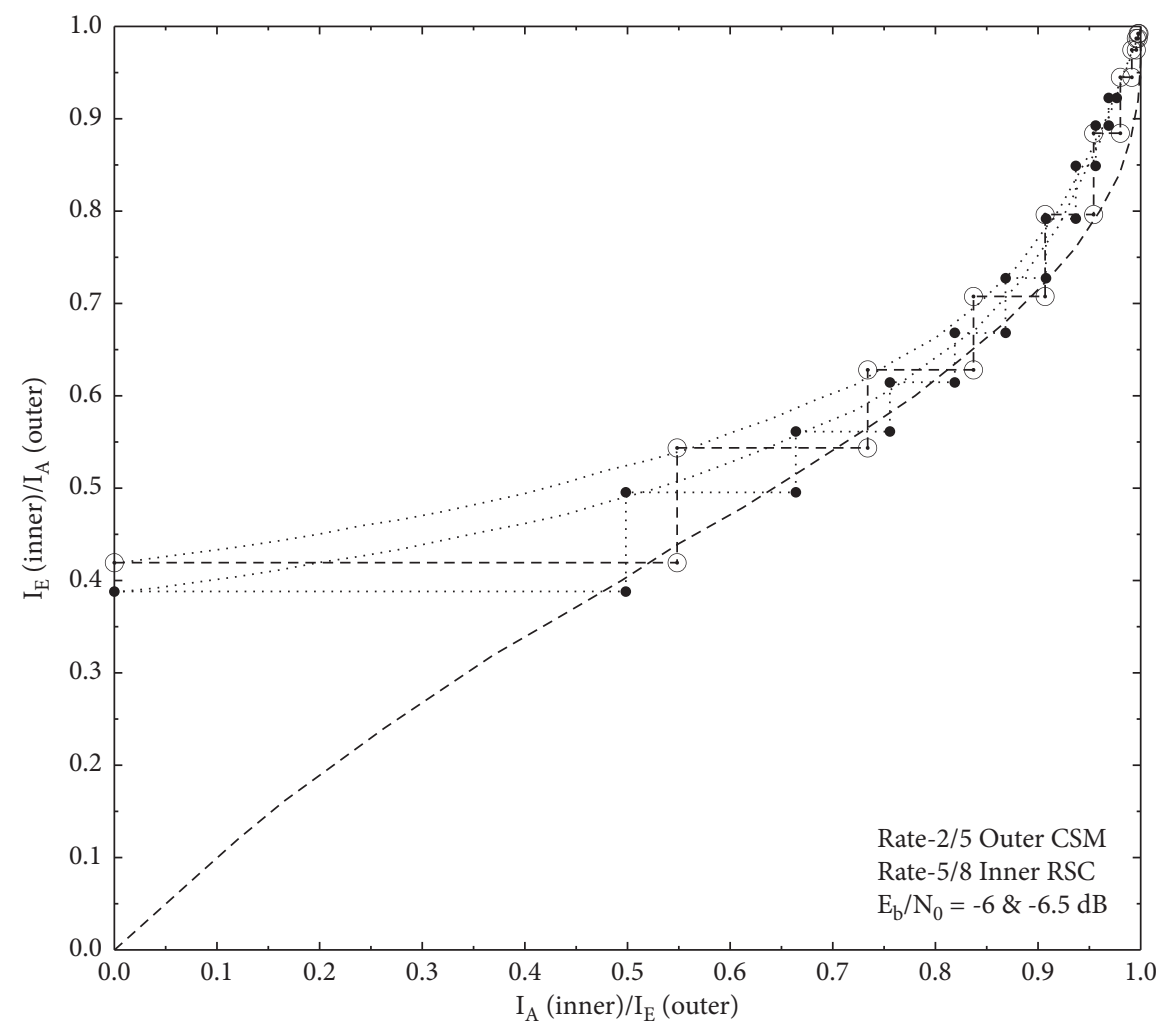

FIGURE 9: EXIT chart curves and decoding trajectories for Rate-4/10 CSM (Mapping-II) scheme.

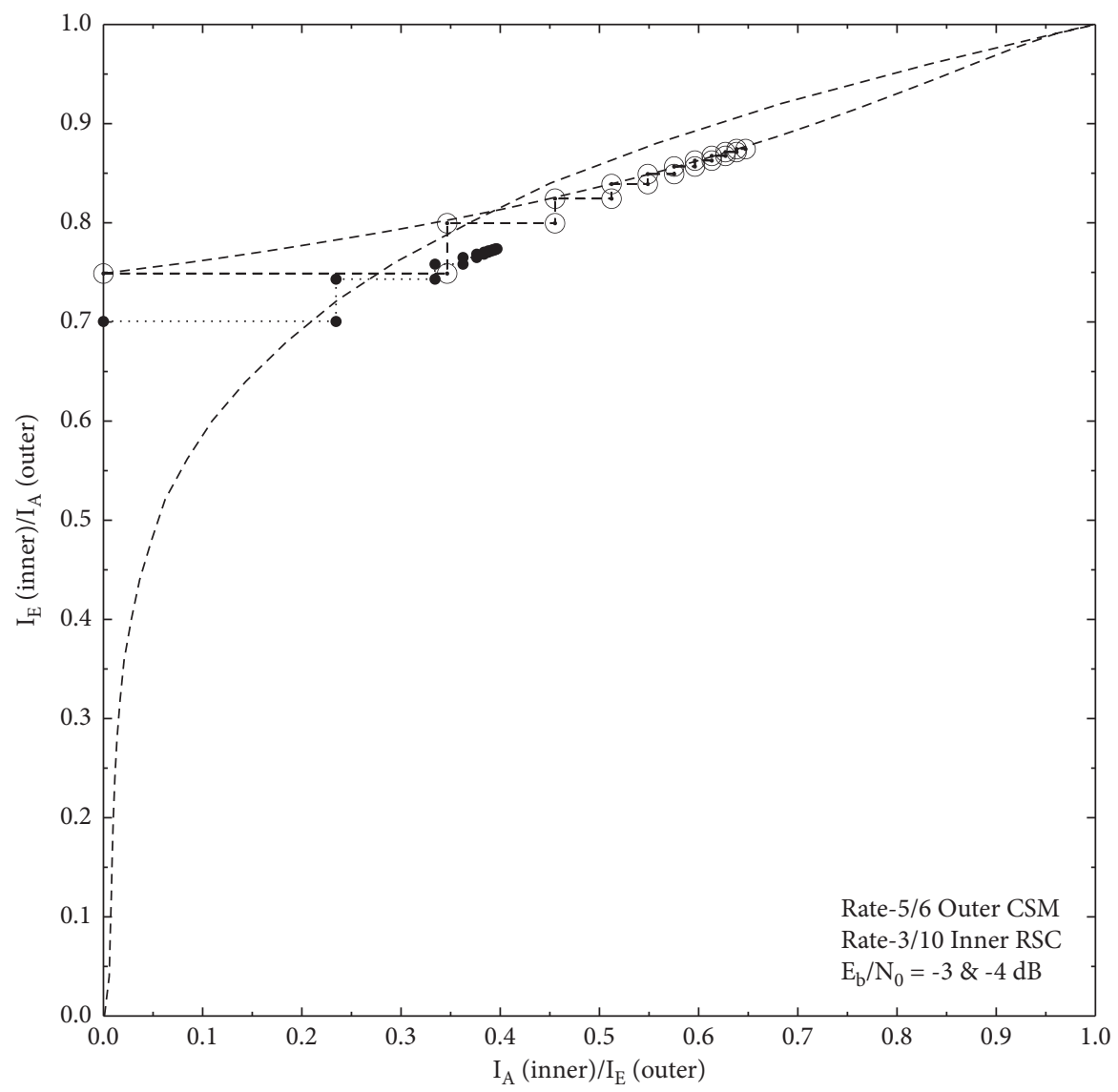

Figure 10: EXIT chart curves and decoding trajectories for Rate-5/6 CSM (Mapping-I) scheme. 


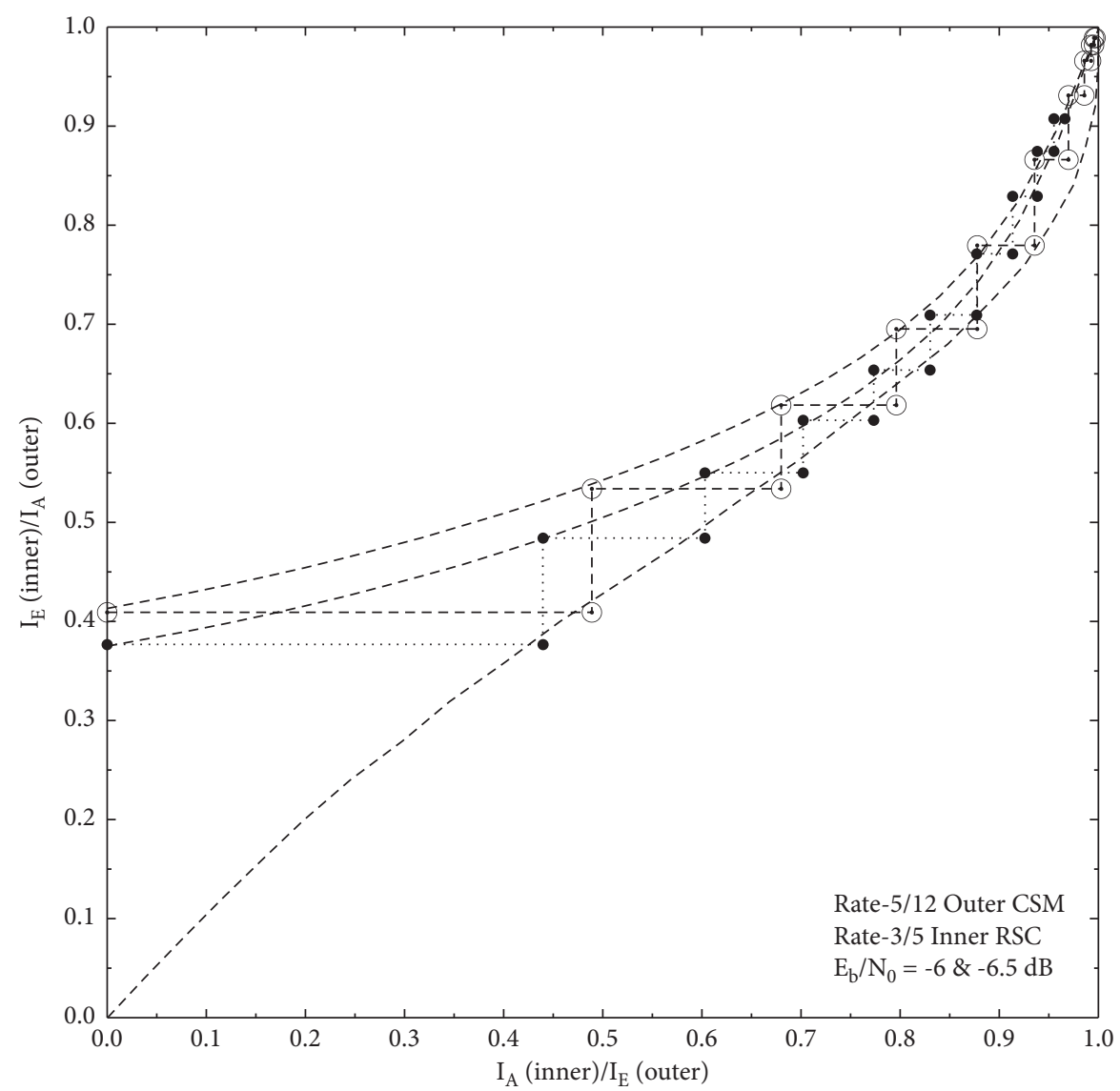

FIgURE 11: EXIT chart curves and decoding trajectories for Rate-5/12 CSM (Mapping-II) scheme.

\section{Proposed System Overview}

Figure 1 depicts the block diagram of our proposed system based on the CSM scheme with DSTS-SP transmission mechanism and utilizing iterative JSCD approach at the receiver. The information bits are first allowed to the H.264 source encoder, transforming the data into squeezed form apt for transmission with minimal essential bandwidth. The compressed stream $x_{k}$ is DeMUltipleXed (DeMUX) to generate three different partitions. The technique of Data Partitioning (DP) is incorporated to assign specific important parameters and coding elements to partitions $\mathrm{A}, \mathrm{B}$, and $\mathrm{C}$. The partitions are concatenated into single stream $x_{i}$. There exists a certain level of unreliability in $x_{i}$, mainly due to the concomitants of compression. To vouch for the desired reliability, CSM encoding is introduced as channel coding technique. CSM encoder transforms the video stream $x_{i}$ into $x_{i}^{\prime}$. The bit-interleaver $\amalg$ assists in boosting the achievable performance of iterative scheme. There is a direct linkage between the degree of statistical independence associated with an interleaver and the number of interleaving bits [39]. The only hitch in longer interleaver is the delay it entails. To cope up with the delay, we concatenated the generated bits such that 99 Macroblocks (MBs) were within each frame. The interleaved stream $\bar{x}_{i}$ is then RSC encoded with a specific rate to yield $y_{i}$, which is SP modulated for propagation over Rayleigh fading channel. Finally, DSTS is applied to revamp the overall gain of system by embracing transmit diversity via two transmitting antennas (Txs).

The decoding operation initiates with single receiving antenna ( $\mathrm{Rx} 1)$. Multiple receiving antennas can be utilized but we proceed with single antenna, merely for easier understanding of the concept. The received signal is allowed to a suboptimum DSTS decoder which continuously accepts interdependent signals from the Txs. The DSTS decoding presents a simple and handy approach for systems benefitting from the MIMO technique. SP demapper brings back the signal to its original frequency range and yields the $L$-values. The next stage constitutes an ISCD between the RSC and SBSD component decoders. The output from RSC decoder is interleaved to serve as a priori input to SBSD. The extrinsic output from SBSD is again deinterleaved $\amalg^{-1}$ and fed to RSC decoder. The iterative process refines and gleans the best possible information exchange between component decoders. The iterative process yields $L\left(\widehat{x}_{m}^{\prime}\right)$, which is passed through CSM decoder to generate the reconstructed stream $\hat{x}_{i}$. The stream is then deconcatenated for forwarding to MUltipleXer (MUX). Eventually, the reconstructed signal $x_{i}$ is processed by the source decoder to produce the decoded bits. 


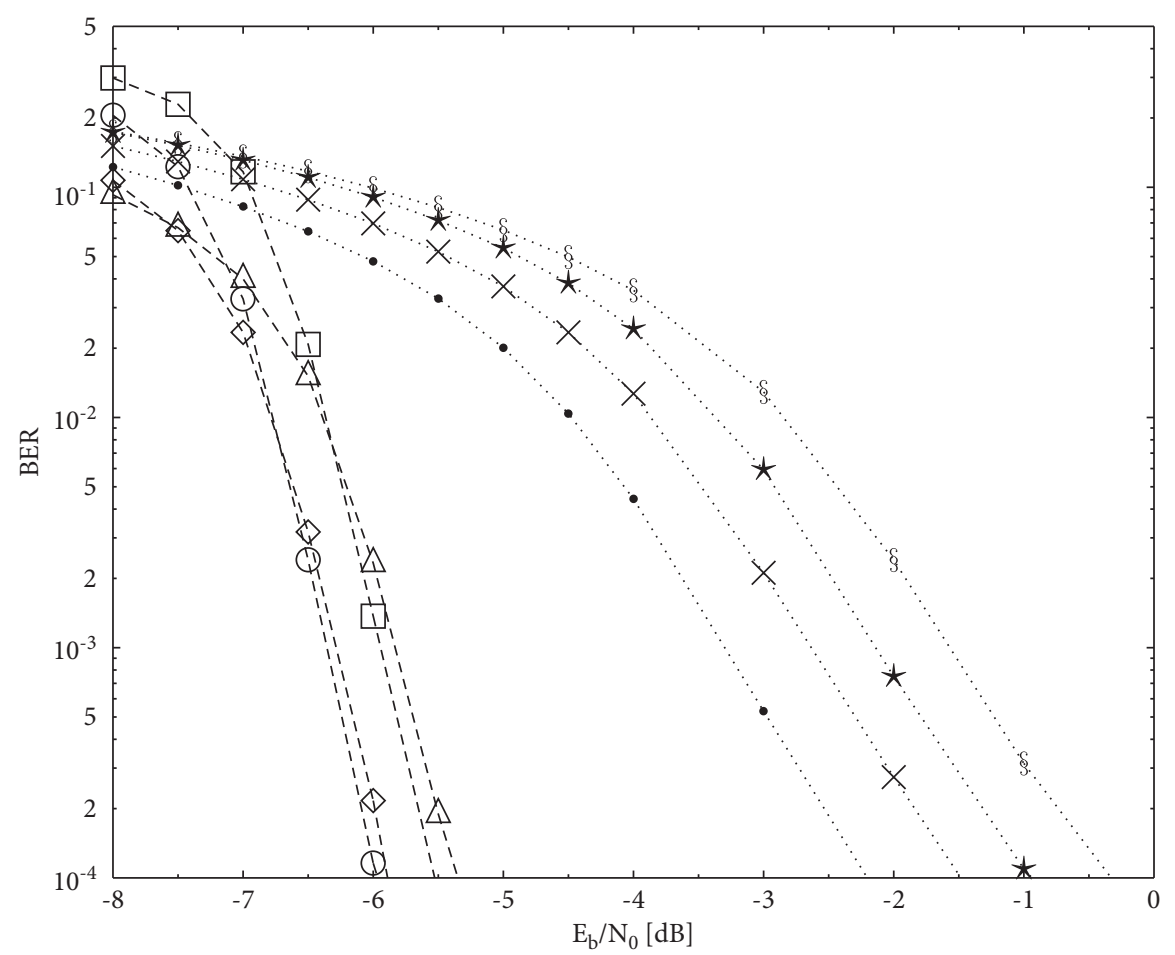

ERROR PROTECTION SCHEMES

\begin{tabular}{|c|c|}
\hline ..... Rate-2/3 CSM & --- Rate-2/6 CSM \\
\hline Rate-3/4 CSM & $\triangle---$ Rate-3/8 CSM \\
\hline Rate- $4 / 5$ CSM & $\square$--- Rate- $4 / 10$ CSM \\
\hline Rate-5/6 CSM & -- Rate-5/12 CSM \\
\hline
\end{tabular}

FIgURE 12: BER performance curves of CSM based schemes presented in Table 3.

\section{Description of the Proposed System}

For the parametric setting stated in Table 1, the proposed system was simulated using the IT++ signal processing and communication library. The Quarter Common Intermediate Format (QCIF) Akiyo is considered as a test video sequence with 15 frames per second $(f p s)$. To even out the effect of error propagation, the encoded video clip complies with the intracoded " $I$ " and predicted " $P$ " frame sequence, placing 44 " $P$ " frames after each " $I$ " frame. A lag of 3 seconds exists between two consecutive " $I$ " frames. Selection of channel model becomes crucial considering the reflection, diffraction, and scattering effects in wireless systems. Such effects result in the multipath phenomenon [40]. Multipath effect splits the transmitted signal into multiple versions based on the power and fading distributions. Rayleigh fading channel is deemed to be the best fitting in such scenario [41] and hence has been considered for our system. The Rayleigh probability density function is given by the following equation as in [41]:

$$
p(z)=\frac{z}{\sigma^{2}} e^{-z^{2} / 2 \sigma^{2}}, \quad z \geq 0 .
$$

Here, $\sigma^{2}=E\left[z^{2}\right]$ is variance of the circularly symmetric complex random variable $z$, having real and imaginary parts. The term $E[*]$ denotes statistical averaging.
DSTS is incorporated in the proposed system to achieve diversity gain. The schematic diagram of DSTS encoder is depicted in Figure 2. The SP modulated signal is differentially encoded till the refined uncorrelated output $q$ is fed to STS encoder. Differentially encoded data is repeatedly looped back until reaching the set value of interleaving delay. The STS encoder governs the spreading of data with the aid of Walsh codes. The overall differentially spread data is divided into two substreams, each transmitted via a specific transmit power antenna.

The CSM process obeys the convergence condition of iterative systems, requiring a minimum Hamming distance $\left(d_{H, \text { min }}\right)$ of at least 2 for valid codewords [42]. The artificial redundancy of CSM assists ISCD in attaining the highest source entropy, given by $H(X)=L_{\mathrm{SBSD}}^{\mathrm{extr}}=1$ bit at the $a$ priori input of $H(X)=L_{\mathrm{SBSD}}^{\mathrm{apri}}=1 \mathrm{bit}$. This leads to the design of CSM schemes known as Mapping-I and Mapping-II, acceding to the condition of $d_{H, \min } \geq 2$.

4.1. Mapping-I. Mapping-I adds single parity in the K-bit information word, yielding $N=(K+1)$-bit CSM-coded symbol and conveniently represented as $\operatorname{CSM}_{K}^{N}$ scheme. The redundant bit can be placed anywhere out of the $(K+1)$ available slots, creating $(K+1)$ different combinations with 

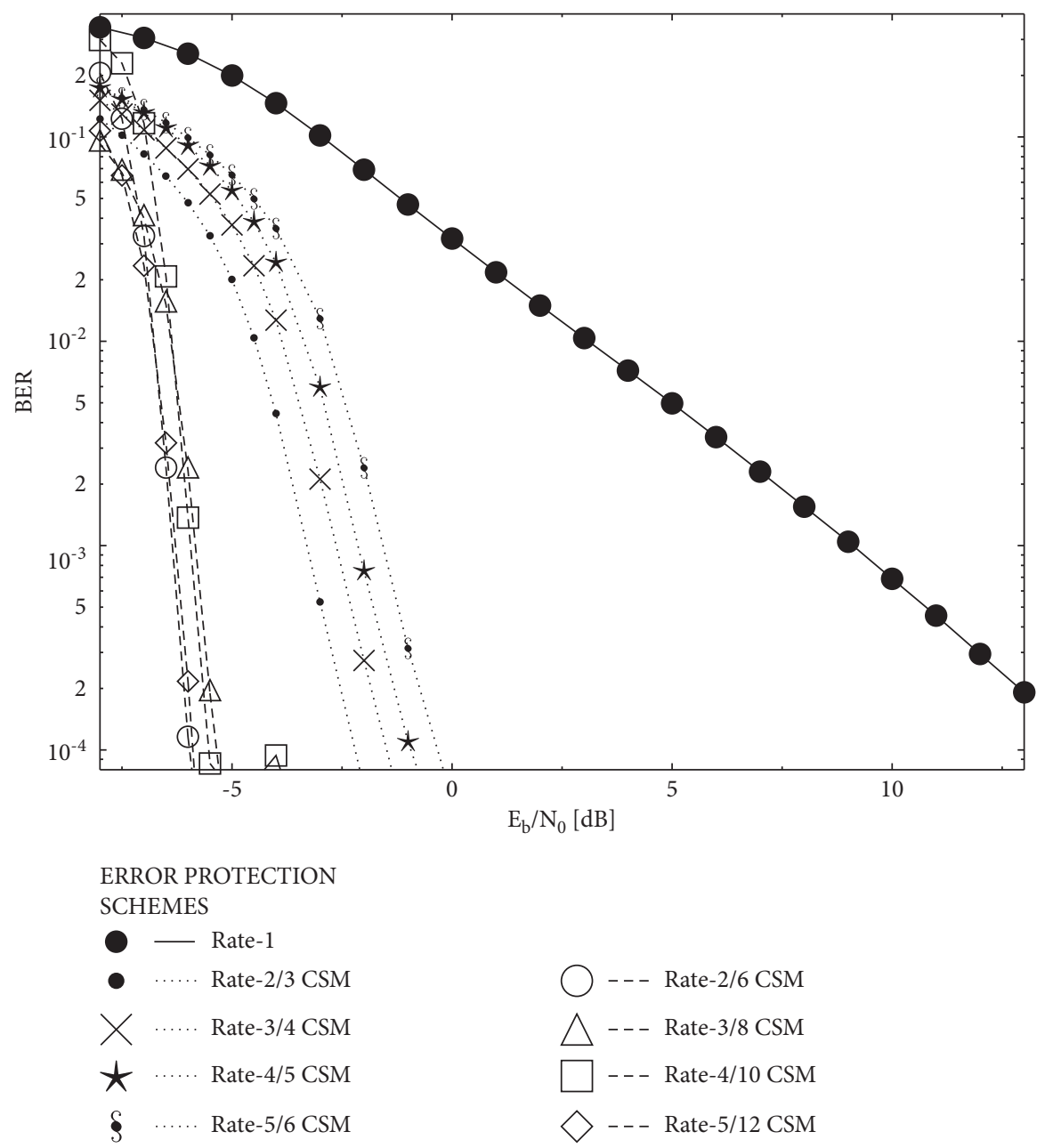

FIGURE 13: BER performance comparison of CSM schemes with Rate-1 benchmarker.

fixed $d_{H, \text { min }}$ of 2 in all. To determine the binary state of generated redundant bit, Mapping-I proceeds by calculating the exclusive OR (XOR) function of the source coded $K$-bit word as in [6].

$$
r_{t}=b_{1}^{t} \oplus b_{2}^{t} \cdots \oplus b_{K}^{t} .
$$

In equation (7), $r_{t}$ represents the redundant bit generated for the $t$-th $M$-ary source word and $\oplus$ denotes the XOR operation between the corresponding input bits.

4.2. Mapping-II. For the sake of increasing $d_{H \text {, min }}$, further $\mathrm{Nbits}$ are appended in a reverse form to the output of Mapping-I. This reduces the code rate of Mapping-II scheme relative to Mapping-I as discussed in [6]. Hence, the output bits in Mapping-II approach will be $(2 \times N)$ bits, where $N=$ $(K+1)$ bits as shown in Table 2 . This can be conveniently written as $\operatorname{CSM}_{K}^{(N \times 2)}$ scheme.

To further elaborate the two schemes, the following symbols as specified by Table 3 make use of a constant overall code rate. It is clearly seen that the developed schemes follow the necessary condition of $d_{H, \min } \geq 2$ all the time except in the case of Rate-1 mapping. For simple understanding, we designate Rate- 1 as the case when no CSM is actually invoked. All the symbols generated using the Mapping-I scheme record $d_{H \text {, min }}$ of 2 , whereas those from Mapping-II have a $d_{H, \text { min }}$ value of 4 . It can be seen that only $2^{K}$ out of $2^{N}$ symbols of Mapping-I and $2^{2 \times N}$ possible symbols of Mapping-II satisfy the $d_{H, \min } \geq 2$ condition. The concatenated RSC encoder provides with the flexibility to cater for the persistent overall code rate. The considered RSC encoder operates by adjusting the puncturing rate to meet the constant bit-rate budget. Puncturing refers to interrupting some bits from transmission to enhance the bandwidth efficiency. The octal format of generator polynomial for the RSC encoder deployed with code memory of 4 is given by $\left(G_{0}, G_{1}, G_{2}, G_{3}=13,15,15,17\right)$.

\section{Results and Discussion}

Our simulations are based on the QCIF Akiyo video test sequence [5] encoded with the H.264/AVC Joint test Model (JM) 19.0 standard. Additionally, the partitioning of each QCIF frame into 9 slices with each slice containing $11 \mathrm{MBs}$ of $16 \times 16$-pixel resolution is considered. Generally, MBs are used in the iteratively decoded systems for enhancing efficiency and assisting the use of larger interleaver lengths [10]. The intraframe MB updates along with DP technique in each 


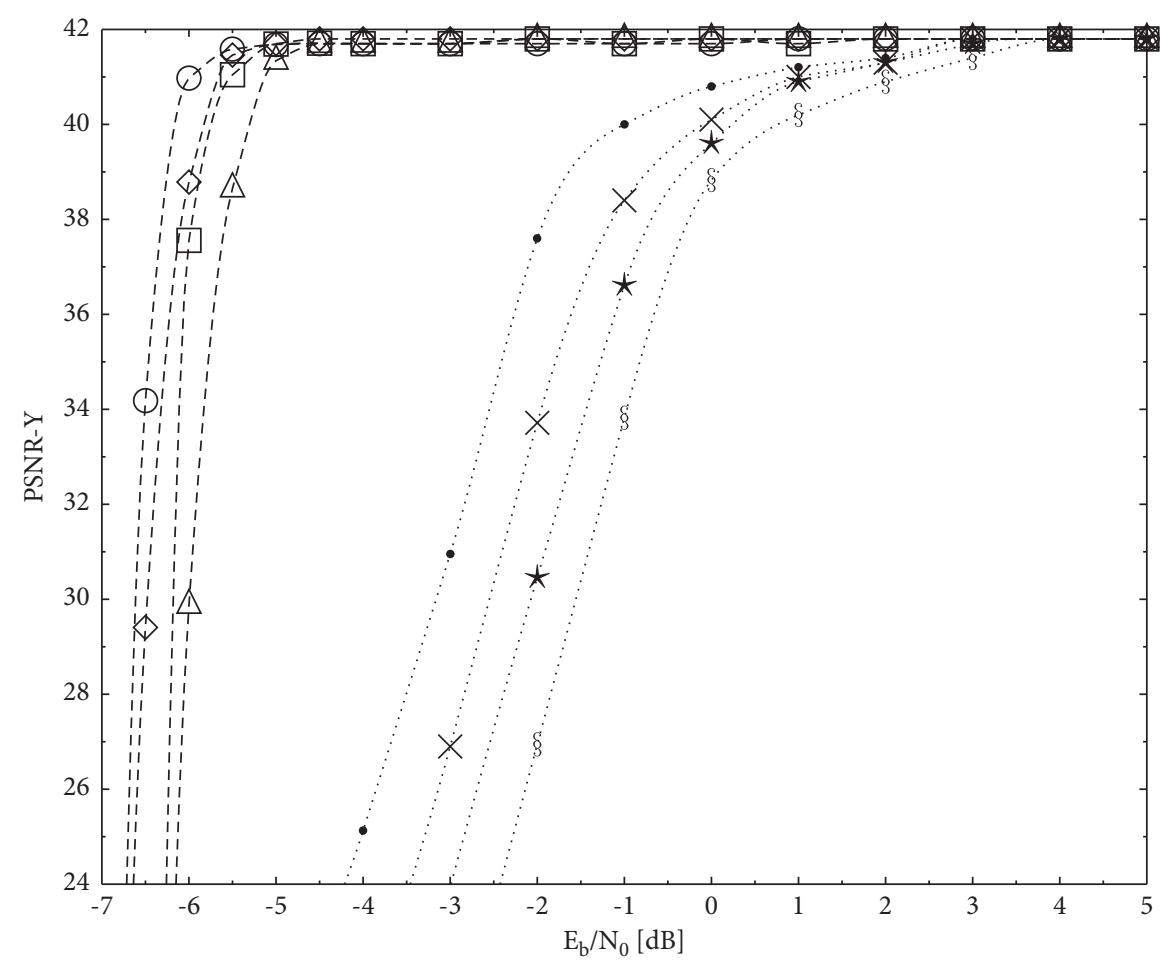

ERROR PROTECTION SCHEMES

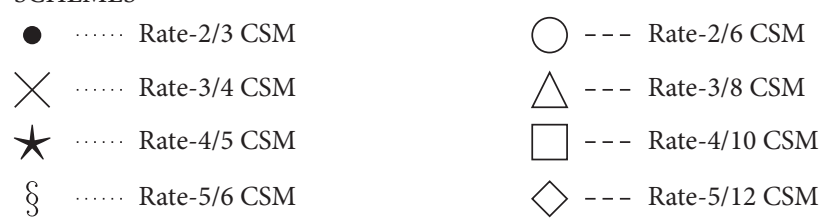

FIGURE 14: PSNR performance curves of CSM based schemes presented in Table 3.

QCIF frame, enhancing the error resiliency of our proposed setup. Flexible MB Ordering (FMO) and interframe prediction using the bidirectional " $B$ " frame were not invoked for the purpose of reduced video coding complexity. However, to better cope with the fluctuations, only the precursor frames were considered. Moreover, for Rate-1 mapping (no artificial redundancy), we limited the number of iterations between the constituent RSC and SBSD decoders to 5 with the intention to limit the computational complexity. However, the number of iterations is set to 10 for other schemes employing CSM with rate less than unity. For enhancing accuracy and confidence in our simulations, we use the average results after repeating the experiment 160 times. It is worthy to state that all the considered error protection schemes are simulated for a persistent overall code rate and video rate.

We first investigate the convergence pattern of different candidates and their possible constituent RSC and CSM rates for a persistent overall code rate of $1 / 4$. Figure 3 illustrates the EXIT characteristics of three types of protection schemes simulated for our proposed iterative DSTS-SP system. It is observable that the incorporation of DSTS-SP approach to CSM technique is favourable for rate less than unity CSM schemes. Explicitly, for such schemes, the iterative SBSD becomes favourable for the EXIT curves to attain $(1,1)$ point of convergence. This allows reliable transmissions for these schemes as the convergence point is responsible for an infinitesimal BER. The system is unable to attain convergence for Rate-1 mapping (i.e., no CSM) as no artificial redundancy is introduced in the source coded stream. More explicitly, the outer EXIT curves associated with Mapping-I and Mapping-II schemes ultimately reach the top-right corner point following different paths as shown in Figure 3.

Further analysis on the corresponding convergent schemes employing Mapping-I and Mapping-II CSM schemes along with the associated RSCs as summarised in Table 3 is done at $E_{b} / N_{0}=-3$ and -4 and $E_{b} / N_{0}=-6$ and $-6.5 \mathrm{~dB}$, respectively. It can be clearly observed that the EXIT chart curves for Mapping-II CSM require lower $E_{b} / N_{0}$ for convergence compared to the Mapping-I CSM. The corresponding EXIT curves along with the decoding trajectories for different CSM rates are portrayed in Figures 4-11. The inner and outer decoders assist in an iterative fashion, sharing mutual information with each other to generate decoding trajectories based on the Monte Carlo simulation algorithm. It is clear from Figures $4-11$ that the reduction of CSM rates positively affects the convergence 


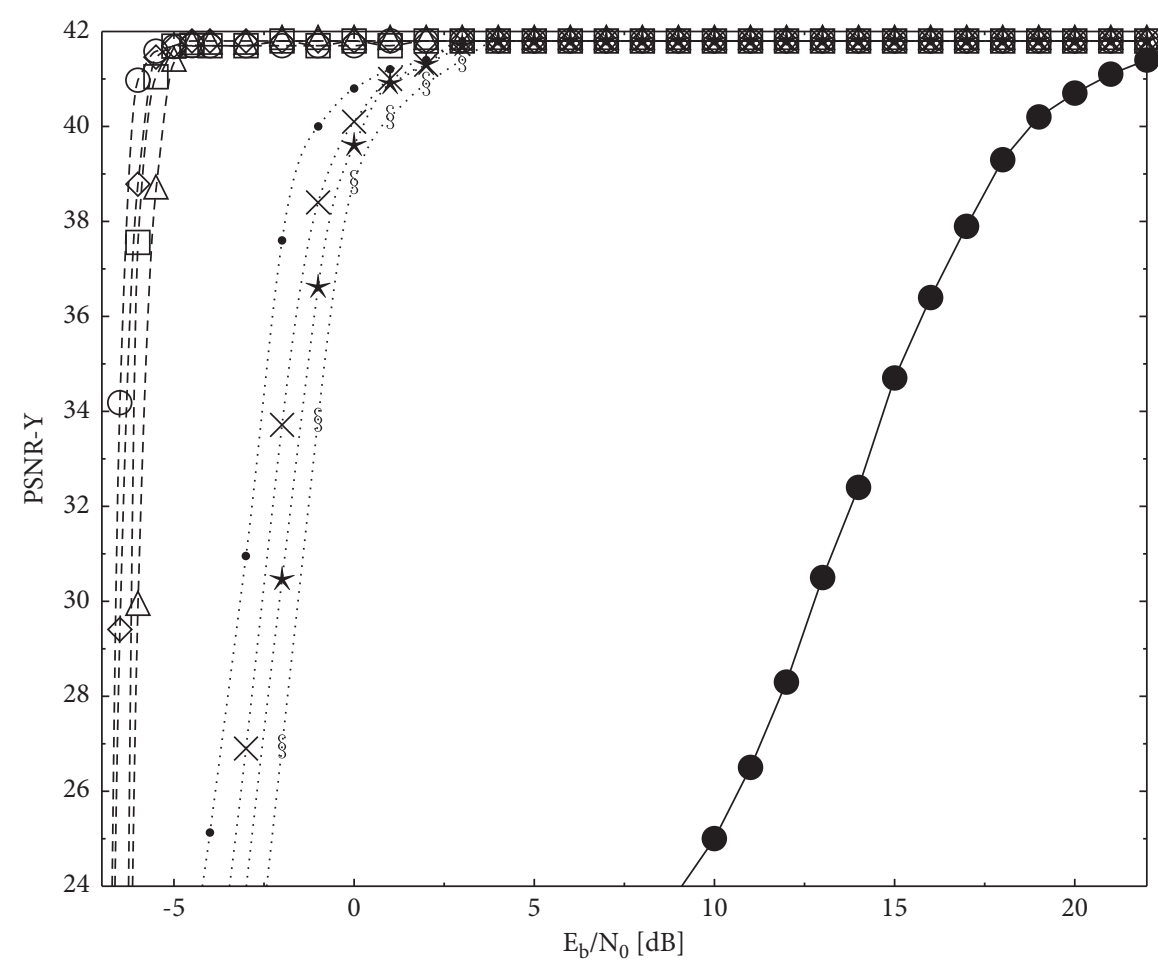

ERROR PROTECTION

SCHEMES
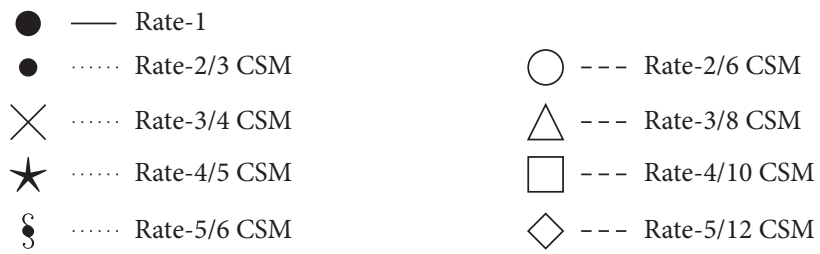

FIGURE 15: PSNR performance comparison of CSM schemes with Rate-1 benchmarker.

pattern of both Mapping-I and Mapping-II schemes. Furthermore, the Mapping-II scheme portrays additional improvement compared to the Mapping-I scheme owing to the improved $d_{H \text {, min }}$ and greater number of redundant bits. The convergence of Mapping-I and Mapping-II schemes is in accordance with the Kliewer criterion as discussed in [42]. In Figures 4-11, the outer and inner EXIT curves intersect each other after the formation of an open tunnel between the two curves. This tunnel is referred to as the convergence tunnel [38], once the constituent curves meet at the $(1,1)$ point of perfect convergence. According to [43], the wider convergence tunnel results in better convergence performance of the schemes. Figures 4-11 confirm that the associated tunnel becomes more favourable in terms of convergence performance when lower CSM rates are invoked. We can see in Figures 8 and 10 that, for the Mapping-I scheme, the outer and inner EXIT curves intersect each other two times. More specifically, here the point of intersection prior to $(1,1)$ point of perfect convergence results in semiconvergent tunnel for Rate-4/5 CSM and Rate-5/6 CSM. This semiconvergent tunnel specifies a moderately lower BER achievement due to convergence rather than an infinitesimal BER at perfect convergence. The Monte Carlo based trajectories accordingly validate the pattern of convergence associated with each of the schemes in Figures 4-11. For capacity analysis, the higher number of iterations between the EXIT curves is associated with a more nearer performance to the corresponding channel's capacity as reported in [38]. Comparing Mapping-I and Mapping-II schemes, it is deducible from Figures 4-11 that Mapping-II scheme requires a greater number of iterations. Hence, Mapping-II performance is more proximal to capacity than Mapping-I performance for the same channel. Similarly, for any of the Mapping-I or Mapping-II scheme, the number of iterations required for convergence is more when the outer CSM rate is least.

The BER performance of different CSM based error protection schemes of Table 3 is depicted in Figure 12. Furthermore, the comparison of these CSM based schemes with Rate-1 is presented in Figure 13. The Peak Signal-toNoise Ratio (PSNR) performance metric of CSM based schemes is represented in Figure 14, while comparison with Rate- 1 scheme is done in Figure 15. Both the BER and PSNR performance curves endorse our claim that the performance of CSM based schemes improves upon reducing the rate of CSM. Furthermore, it is clearly visible from Figures 12-15 

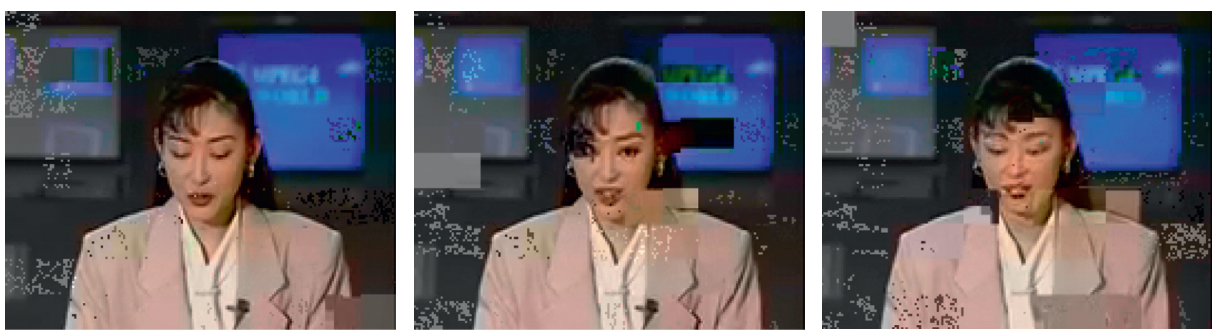

(a)
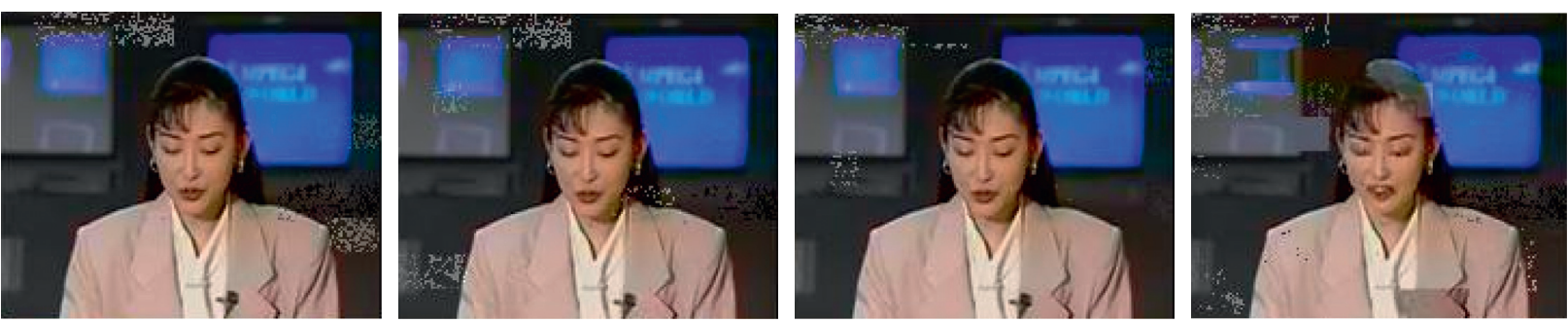

(b)

Figure 16: Subjective video quality performance of (a) Mapping-I and (b) Mapping-II CSM schemes at $E_{b} / N_{0}=-2.5 \mathrm{~dB}$ and $-6 \mathrm{~dB}$, respectively.

that the BER and PSNR performances improve with the increase in $d_{H, \text { min }}$ value for the advocated CSM schemes. More specifically, for Rate-1 scheme, the outer and inner EXIT curves do not converge and, therefore, an infinitesimally lower BER cannot be attained. For the PSNR curves shown in Figure 15, the best PSNR is provided by the Rate-2/ 6 CSM invoked with Rate-3/4 inner RSC. To be precise, up to $20 \mathrm{~dB}$ gain in $E_{b} / N_{0}$ is recorded with the CSM scheme in comparison to the benchmarker.

Lastly, the subjective video quality performance of Mapping-I and Mapping-II CSM schemes for the 45th Akiyo video sequence is given by Figure 16. The top frames from left to right are recorded at $E_{b} / N_{0}=-2.5 \mathrm{~dB}$ for Rate-2/3, Rate-3/4, Rate-4/5, and Rate-5/6 Mapping-I CSM schemes, respectively. The bottom frames from left to right are recorded at $E_{b} / N_{0}=$ $-6 \mathrm{~dB}$ for Rate-2/6, Rate-3/8, Rate-4/10, and Rate-5/12 Mapping-II CSM schemes, respectively. The video test sequence was separately transmitted for each type of scheme presented and was accordingly decoded via the H.264 decoder. It is clear from Figure 16 that the subjective video quality also holds the claim of improved performance for lower CSM rates. Visibly, there are considerably more distortions in Mapping-I schemes even at higher $E_{b} / N_{0}$ in comparison to Mapping-II schemes. Additionally, employing Mapping-II CSM scheme offers even more superior video quality at considerably lower value of $-6 \mathrm{~dB}$ relative to Mapping-I CSM.

\section{Conclusion}

The overall system developed offers significant performance improvements; hence, it will be beneficial in wireless communication technologies. The proposed system is an attempt to improve the PSNR and BER performances using the approach of DSTS-SP-assisted smart antennas which requires no CSI for the decoding process. The CSM technique was used to incorporate artificial redundancy in the
SBSD-aided H.264/AVC standard. The novel CSM technique offers a beneficial way to flexibly change the $d_{H \text {, min }}$ value accordingly for an enhanced and reliable performance. Furthermore, CSM is divided into two schemes based on the generation and number of artificial redundant bits. The BER performance of DSTS-SP-assisted ISCD was revealed to significantly improve by incorporating rate $<1$ CSM and favourably dividing the bit-rate budget between source and channel codes. EXIT chart analysis also recommends our proposed system as it indicates fruitful convergence patterns. The simulation results clearly advocate for the proposed system. More specifically, the DP H.264 video codec with Rate-2/6 CSM and $d_{H \text {, min }}=4$ evinces an $E_{b} / N_{0}$ gain of $5 \mathrm{~dB}$ relative to Rate $-2 / 3 \mathrm{CSM}$ and $d_{H \text {, min }}=2$, at the PSNR degradation point of $2 \mathrm{~dB}$. Additionally, the presented system with Rate-2/3 CSM scheme outperforms the benchmarker (no CSM) scheme having persistent overall rate by about $20 \mathrm{~dB}$. In the future, we aspire to subsume the approach of DSTS in other modulation techniques and stateof-the-art multimedia applications.

\section{Data Availability}

The data used to support the findings of this study are embedded in the programming. They are available from the corresponding author upon request.

\section{Conflicts of Interest}

The authors declare that there are no conflicts of interest regarding the publication of this study.

\section{Acknowledgments}

The financial support of National Centre in Big Data and Cloud Computing, University of Engineering and 
Technology Peshawar (NCBC-UETP), under the auspices of Higher Education Commission, Pakistan, is gratefully acknowledged. The authors would like to thank and acknowledge the VSB-Technical University of Ostrava and the Ministry of Education, Youth and Sports in the Czech Republic for funding the publication charges of this article (Project nos. SP2021/45 and SP2021/32).

\section{References}

[1] F. Cogen, E. Aydin, N. Kabaoglu, B. Ertugrul, and H. Ilhan, "Generalized code index modulation and spatial modulation for high rate and energy-efficient MIMO systems on Rayleigh block-fading channel," IEEE Systems Journal, vol. 15, no. 1, 2021.

[2] L. Hanzo, O. Alamri, M. El-Hajjar, and N. Wu, Near-capacity multi-functional MIMO systems: sphere-packing, iterative detection and cooperation, Vol. 4, John Wiley \& Sons, Hoboken, NJ, USA, 2009.

[3] X. Yang, D. Fan, A. Ren, N. Zhao, and M. Alam, "5G-based user-centric sensing at C-band," IEEE Transactions on Industrial Informatics, vol. 15, no. 5, pp. 3040-3047, 2019.

[4] K. Arora, J. Singh, and Y. S. Randhawa, "A survey on channel coding techniques for $5 \mathrm{G}$ wireless networks," Telecommunication Systems, vol. 73, no. 4, pp. 637-663, 2020.

[5] L. Hanzo, C. Peter, and J. Streit, Video Compression And Communications: From Basics to H. 261, H. 263, H. 264, MPEG4 for DVB and HSDPA-Style Adaptive Turbo-Transceivers, John Wiley \& Sons, Hoboken, NJ, USA, 2007.

[6] L. Hanzo, "EXIT-chart optimized short block codes for iterative joint source and channel decoding in H. 264 video telephony," IEEE Transactions on Vehicular Technology, vol. 58, no. 8, pp. 4306-4315, 2009.

[7] T. Fingscheidt and P. Vary, "Softbit speech decoding: a new approach to error concealment," IEEE Transactions on Speech and Audio Processing, vol. 9, no. 3, pp. 240-251, 2001.

[8] M. Adrat and P. Vary, "Iterative source-channel decoding: improved system design using EXIT charts," EURASIP Journal on Applied Signal Processing, vol. 2005, no. 6, Article ID 178541, 2005.

[9] W.-Y. Kung, C.-S. Kim, and C.-C. J. Kuo, "Spatial and temporal error concealment techniques for video transmission over noisy channels," IEEE Transactions on Circuits and Systems for Video Technology, vol. 16, no. 7, pp. 789-803, 2006.

[10] T. Stockhammer, M. M. Hannuksela, and T. Wiegand, "H.264/AVC in wireless environments," IEEE Transactions on Circuits and Systems for Video Technology, vol. 13, no. 7, pp. 657-673, 2003.

[11] R. G. Maunder, J. Wang Soon Xin Ng, S. X. Ng, L.-L. Yang, and L. Hanzo, "On the performance and complexity of irregular variable length codes for near-capacity joint source and channel coding," IEEE Transactions on Wireless Communications, vol. 7, no. 4, pp. 1338-1347, 2008.

[12] C. E. Shannon, "A mathematical theory of communication," Bell System Technical Journal, vol. 27, no. 3, pp. 379-423, 1948.

[13] M. Z. Chowdhury, M. Shahjalal, S. Ahmed, and Y. M. Jang, "6G wireless communication systems: applications, requirements, technologies, challenges, and research directions," IEEE Open Journal of the Communications Society, vol. 1, pp. 957-975, 2020.

[14] S. M. Alamouti, "A simple transmit diversity technique for wireless communications," IEEE Journal on Selected Areas in Communications, vol. 16, no. 8, pp. 1451-1458, 1998.
[15] V. Tarokh, H. Jafarkhani, and A. R. Calderbank, "Space-time block codes from orthogonal designs," IEEE Transactions on Information Theory, vol. 45, no. 5, pp. 1456-1467, 1999.

[16] B. Hochwald, T. L. Marzetta, and C. B. Papadias, "A transmitter diversity scheme for wideband CDMA systems based on space-time spreading," IEEE Journal on Selected Areas in Communications, vol. 19, no. 1, pp. 48-60, 2001.

[17] W. Su, Z. Safar, and K. J. R. Liu, "Space-time signal design for time-correlated Rayleigh fading channels,"vol. 5, pp. 31753179, in In Proceedings of the IEEE International Conference on Communications, 2003. ICC'03, vol. 5, IEEE, Anchorage, AK, USA, May, 2003.

[18] M. El-Hajjar, O. Alamri, S. X. Soon Xin Ng, and L. Hanzo, "Turbo detection of precoded sphere packing modulation using four transmit antennas for differential space-time spreading," IEEE Transactions on Wireless Communications, vol. 7, no. 3, pp. 943-952, 2008.

[19] V. Tarokh and H. Jafarkhani, "A differential detection scheme for transmit diversity," IEEE Journal on Selected Areas in Communications, vol. 18, no. 7, pp. 1169-1174, 2000.

[20] H. Jafarkhani and V. Tarokh, "Multiple transmit antenna differential detection from generalized orthogonal designs," IEEE Transactions on Information Theory, vol. 47, no. 6, pp. 2626-2631, 2001.

[21] N. S. Othman, M. El-Hajjar, O. Alamri, S. X. Ng, and L. Hanzo, "Iterative AMR-WB source and channel decoding using differential space-time spreading-assisted spherepacking modulation," IEEE Transactions on Vehicular Technology, vol. 58, no. 1, pp. 484-490, 2008.

[22] M. El-Hajjar, R. G. Maunder, O. Alamri, S. X. Ng, and L. Hanzo, Iteratively detected irregular variable length coding and sphere-packing modulation-aided differential space-time spreading," in Proceedings of the 2007 IEEE 66th Vehicular Technology Conference, pp. 1238-1242, IEEE, Baltimore, MD, USA, Octobar, 2007.

[23] N. Minallah, K. Ullah, J. Frnda, K. Cengiz, and M. Awais, "Transmitter diversity gain technique aided irregular channel coding for mobile video transmission," Entropy, vol. 23, no. 2, p. 235, 2021.

[24] S. Sugiura, S. Chen, and L. Hanzo, "Cooperative differential space-time spreading for the asynchronous relay aided CDMA uplink using interference rejection spreading code," IEEE Signal Processing Letters, vol. 17, no. 2, pp. 117-120, 2009.

[25] N. Minallah, I. Ahmed, M. Ijaz, A. Sardar Khan, L. Hasan, and A. Rehman, "On the performance of self-concatenated coding for wireless mobile video transmission using DSTS-SPassisted smart antenna system," Wireless Communications and Mobile Computing, vol. 2021, Article ID 8836808, 10 pages, 2021.

[26] N. Minallah, K. Ullah, J. Frnda, L. Hasan, and J. Nedoma, “On the performance of video resolution, motion and dynamism in transmission using near-capacity transceiver for wireless communication," Entropy, vol. 23, no. 5, p. 562, 2021.

[27] N. Minallah, I. Ahmed, J. Frnda, and K. S. Khattak, "Averting BER floor with iterative source and channel decoding for layered steered space-time codes," Sensors, vol. 21, no. 19, p. $6502,2021$.

[28] A. Khalil, N. Minallah, I. Ahmed, K. Ullah, J. Frnda, and N. Jan, "Robust mobile video transmission using DSTS-SP via three-stage iterative joint source-channel decoding," Humancentric Computing and Information Sciences, vol. 11, 2021.

[29] J. Hagenauer, E. Offer, and L. Papke, "Iterative decoding of binary block and convolutional codes," IEEE Transactions on Information Theory, vol. 42, no. 2, pp. 429-445, 1996. 
[30] L. Lu, G. Y. Li, A. L. Swindlehurst, A. Ashikhmin, and R. Zhang, "An overview of massive MIMO: benefits and challenges," IEEE journal of selected topics in signal processing, vol. 8, no. 5, pp. 742-758, 2014.

[31] C. K. Ho, J. H. Cheong, J. Lee et al., "High bandwidth efficiency and low power consumption Walsh code implementation methods for body channel communication," IEEE Transactions on Microwave Theory and Techniques, vol. 62, no. 9, pp. 1867-1878, 2014.

[32] S. Wolfram, A new kind of science, Vol. 5, Wolfram media, Champaign, IL, USA, 2002.

[33] R. W. Hamming, "Error detecting and error correcting codes," Bell System Technical Journal, vol. 29, no. 2, pp. 147-160, 1950.

[34] P. Chen, Y. Wang, Q. Yu, Y. Fan, and R. Feng, "Hamming distance encoding multihop relation knowledge graph completion," IEEE Access, vol. 8, Article ID 117146, 2020.

[35] T. B. Stephan, "Designing iterative decoding schemes with the extrinsic information transfer chart," AEU International Journal Electronic Communication, vol. 54, no. 6, pp. 389-398, 2000.

[36] S. Bhattacharjee, M. Damrath, and P. A. Hoeher, "EXIT chart analysis of higher order modulation schemes in molecular communications," in Proceedings of the 6th Annual ACM International Conference on Nanoscale Computing and Communication, pp. 1-6, New York, USA, September, 2019.

[37] N. Minallah, M. F. U. Butt, I. U. Khan et al., "Analysis of nearcapacity iterative decoding schemes for wireless communication using EXIT charts," IEEE Access, vol. 8, Article ID $124424,2020$.

[38] L. Hanzo, M. El-Hajjar, and O. Alamri, "Near-capacity wireless transceivers and cooperative communications in the MIMO era: evolution of standards, waveform design, and future perspectives," Proceedings of the IEEE, vol. 99, no. 8, pp. 1343-1385, 2011.

[39] R. Maunder, J. Kliewer, S. Ng, L.-L. Wang, and L. Hanzo, "Joint iterative decoding of trellis-based VQ and TCM," IEEE Transactions on Wireless Communications, vol. 6, no. 4, pp. 1327-1336, 2007.

[40] J. Li, A. Bose, and Q. Yiqiang, "Rayleigh flat fading channels" capacity," in Proceedings of the 3rd Annual Communication Networks and Services Research Conference (CNSR'05), pp. 214-217, IEEE, Halifax, NS, Canada, May, 2005.

[41] M. Divya, "Bit error rate performance of bpsk modulation and ofdm-bpsk with Rayleigh multipath channel," International Journal of Engineering and Advanced Technology, vol. 2, no. 4, pp. 623-626, 2013.

[42] J. Kliewer, N. Goertz, and A. Mertins, "Iterative sourcechannel decoding with Markov random field source models," IEEE Transactions on Signal Processing, vol. 54, no. 10, pp. 3688-3701, 2006.

[43] F. Cheng, A. Liu, Q. Zhang, Y. Zhang, and B. Cai, "Codes design based on EXIT chart for polar coded BICM-ID," in Proceedings of the 2017 IEEE 2nd Advanced Information Technology, Electronic and Automation Control Conference (IAEAC), pp. 1129-1133, IEEE, Chongqing, China, March, 2017. 